\title{
Philosophiques
}

\section{Le moment normatif dans la philosophie austro-allemande}

\section{Guillaume Fréchette}

Volume 42, numéro 2, automne 2015

URI : https://id.erudit.org/iderudit/1034747ar

DOI : https://doi.org/10.7202/1034747ar

Aller au sommaire du numéro

Éditeur(s)

Société de philosophie du Québec

ISSN

0316-2923 (imprimé)

1492-1391 (numérique)

Découvrir la revue

Citer ce document

Fréchette, G. (2015). Le moment normatif dans la philosophie austro-allemande. Philosophiques, 42(2), 375-384.

https://doi.org/10.7202/1034747ar d'utilisation que vous pouvez consulter en ligne.

https://apropos.erudit.org/fr/usagers/politique-dutilisation/ 


\title{
Le moment normatif dans la philosophie austro- allemande
}

\author{
GUILLAUME FRÉCHETTE \\ Université de Genève et Université de Salzbourg
}

\section{Le principe Husserl-Mulligan}

Une opposition centrale entre deux approches du langage et de l'esprit traverse l'ouvrage de Kevin Mulligan: l'approche wittgensteinienne, selon laquelle la philosophie de l'esprit formule des règles sur l'usage des mots, et l'approche consistant à formuler des vérités nécessaires ou essentielles au sujet de l'esprit, une approche représentée particulièrement par la tradition brentanienne. Mulligan suggère de voir les descriptions wittgensteiniennes comme des fragments s'insérant dans le système de descriptions mis de l'avant dans la psychologie descriptive brentanienne. Suivant Mulligan, on peut voir l'ensemble des descriptions brentaniennes comme un système, non pas de règles, mais d'ensembles qui correspondent à des règles et qui justifient ces règles ${ }^{1}$. Sur cette base, on peut alors montrer que la contribution de Wittgenstein à la philosophie austro-allemande est aussi une contribution au projet de Brentano. Cette suggestion de Mulligan (SM) n'est certainement pas wittgensteinienne, mais elle n'est pas incompatible avec certaines conceptions des héritiers de Brentano, même si elle n'est pas vraiment défendue par ces derniers.

Cette suggestion - considérer la psychologie descriptive brentanienne comme un système de règles, dont certaines sont les règles de Wittgenstein et où toutes les règles sont en partie justifiées par des vérités essentielles, ou (SM) — se base sur deux éléments :

Le premier est la thèse descriptive de Mulligan, étayée tout au long du livre, selon laquelle plusieurs des observations communes de Wittgenstein et des héritiers de Brentano sont comprises d'un côté par Wittgenstein comme des expressions de normes et de l'autre côté par les héritiers de Brentano comme des vérités essentielles (TM).

Or (TM) et (SM) présupposent que les phrases qui peuvent être lues comme l'expression de vérités essentielles peuvent aussi fonctionner comme des règles (tout en n'étant pas des règles). C'est là le deuxième élément, qui est un principe central de l'ouvrage de Mulligan, qu'on appellera ici le principe Husserl-Mulligan (HM) :

- $(\mathrm{HM})$ : Les phrases qui expriment des vérités logiques et des vérités essentielles peuvent fonctionner comme des règles.

1. Comparer avec Mulligan (20I2), p. 225. 
Ainsi, la vérité essentielle exprimée par

(I) le vouloir dire n'est pas un processus mental peut fonctionner comme une règle qui pourrait être exprimée ainsi :

(2) il ne faut pas penser que le vouloir dire est un processus mental.

$\mathrm{Si}(2)$ est vrai, alors en partie parce que (I) ${ }^{2}$.

Le cas de (I) et de (2) est une application du principe (HM) à une vérité essentielle concernant le vouloir dire, mais il ne signifie pas que (HM) est limité au cas du vouloir dire. La formulation de (HM) est une extension de deux idées centrales des Prolégomènes de Husserl: la première est celle que les normes sont partiellement fondées dans des vérités logiques:

toute discipline normative, tout comme toute discipline pratique [repose] sur une ou plusieurs disciplines théoriques [...], dans la mesure où ses règles doivent posséder un contenu théorique séparable de l'idée de la normalisation (du devoir [Sollen]), et dont l'étude scientifique incombe précisément à ces disciplines théoriques ${ }^{3}$.

La seconde idée découle de la première:

les supposés axiomes logiques ne sont pas en soi des normes, c'est plutôt qu'ils servent de normes ${ }^{4}$.

Dans son intervention lors de la table ronde puis dans des échanges ultérieurs, Mulligan proposait d'exemplifier le principe (HM) de la manière suivante pour les vérités logiques:

I) $(\mathrm{p} \&(\mathrm{p} \rightarrow \mathrm{q})) \rightarrow \mathrm{q}$

2) si $(\mathrm{p} \&(\mathrm{p} \rightarrow \mathrm{q}))$, alors on a le droit d'inférer à $\mathrm{q}$

3) si (2), alors (2) en partie parce que (I)

On pourrait élargir cette exemplification en l'appliquant non seulement aux normes, mais aussi à l'évidence, disons (HM*):

I) $(\mathrm{p} \&(\mathrm{p} \rightarrow \mathrm{q})) \rightarrow \mathrm{q}$

2) il est évident que (I)

3) si (2), alors (2) en partie parce que (I)

Un élargissement semblable est envisageable pour les vérités essentielles non logiques (HM*e):

I) toute ce qui est coloré est étendu

2) il est évident que (I)

3) si $x$ est coloré, alors on a le droit d'inférer que $x$ est étendu

4) si (3), alors (3) en partie parce que (I)

5) si (2), alors (2) en partie parce que (I)

2. Formulation inspirée de Mulligan (20I2), p. I $5 s q$.

3. Husserl (I900/1959), p. 40/45. Traduction française modifiée.

4. Husserl (I900), p. I 58. 
$\left(\mathrm{HM}^{*}\right)$ va dans le sens de la conception husserlienne de l'évidence, et rien n'indique que Mulligan refuserait cette application:

nous ne doutons pas [...] du fait que la discipline pratique de la logique [logische Kunstlehre] doive étudier les conditions psychologiques dans lesquelles l'évidence nous éclaire dans le jugement [...] et même si nous voulons encore faire la différence entre des propositions purement logiques et des propositions méthodologiques, nous accordons aux premières une certaine relation au donné psychologique de l'évidence et nous leur accordons également qu'elles énoncent en un certain sens les conditions psychologiques de ce donné. Mais nous croyons que cette relation est purement idéale et indirecte. [...] Nous croyons pouvoir montrer que [les propositions purement logiques] ne peuvent entrer en relation avec les vécus d'évidence que par une application ou une transposition [Umwen$d u n g]$, c'est-à-dire de la même manière que chaque loi «purement fondée dans les concepts» peut être transposée sur le domaine [Bereich] représenté de manière générale des cas empiriques individuels de ces concepts ${ }^{5}$.

En d'autres termes, l'évidence des lois logiques — par exemple celle de $(\mathrm{P} \vee \sim \mathrm{P})$ dans une logique bivalente - est une relation «idéale et indirecte » avec les propositions purement logiques: le fait qu'idéalement les lois logiques devraient être évidentes n'implique pas qu'elles le sont, et lorsqu'elles le sont, le fait qu'elles le soient résulte du même genre d'" application ou de transposition " (Umwendung) que lorsque les lois logiques sont instanciées par des cas concrets (les actes mentaux correspondants). Bref, que ces lois puissent être instanciées ou qu'elles puissent être évidentes n'est aucunement constitutif de ce que sont les lois logiques ${ }^{6}$. Je reviendrai sur ce point un peu plus loin.

\section{La reconstruction de l'histoire de la philosophie austro-allemande}

(HM), (TM) et (SM) sont employés par Mulligan dans l'ouvrage principalement pour montrer comment la contribution de Wittgenstein peut être vue comme une contribution au programme de Brentano. Si on accepte cette idée, on doit aussi idéalement accepter une version forte de (TM) selon laquelle non seulement plusieurs des observations des héritiers de Brentano sont comprises comme des vérités essentielles, mais aussi selon laquelle ces observations, partagées par Wittgenstein, sont au centre du programme de Brentano $^{7}$. Accepter la version forte de (TM) a l'avantage de permettre de traiter des théories aussi différentes que la théorie normative de la logique de Brentano, le psychologisme de certains étudiants de Brentano, la théorie de l'objet, la phénoménologie, la théorie martyienne de la signification, l'on-

5. Ibid., p. I 86. Traduction française G. F.

6. On retrouve à peu près la même considération appliquée aux lois essentielles chez plusieurs élèves de Husserl, notamment Reinach (I989) [I9I4], p. 542 [p. 393] sq.

7. Si on ne l'accepte pas, cela voudrait dire que la contribution de Wittgenstein au programme de Brentano serait strictement locale ou accessoire. Il est clair que Mulligan n'entérine pas cette lecture. 
tologie du Tractatus de Wittgenstein, la théorie de la signification comme usage de Wittgenstein, mais aussi, et du même coup, toute une panoplie de théories philosophiques kakaniennes de manière unifiée, sans pour cela avoir recours de manière décisive à des facteurs externes d'unité, comme l'appartenance scolaire, le contexte géographique, ou autre. Pour résumer cette approche de l'histoire de la philosophie austro-allemande, on pourrait dire qu'elle montre que le facteur unifiant de ce mouvement est d'abord et avant tout celui de la description de connexions nécessaires qui rendent vraies les vérités essentielles. L'appartenance scolaire, le contexte géographique, ou encore le rejet du kantisme sont dès lors à voir comme des phénomènes d'importance secondaire qui contribuent naturellement à l'unité du mouvement mais qui ne la définissent pas à proprement parler.

Accepter la version forte de (TM) a l'avantage d'offrir un facteur unifiant fort pour décrire l'unité de la philosophie austro-allemande. Or, malgré son unité, la philosophie austro-allemande est traversée par des conflits doctrinaires relativement importants. On peut penser aux débats sur le psychologisme opposant entre eux plusieurs héritiers de Brentano (Husserl et Meinong d'un côté, Brentano, Kraus, Kastil, de l'autre), sur le transcendantalisme (Husserl le Jeune, Brentano, Meinong, Stumpf, Marty, etc. d'un côté, et Husserl le Vieux et en partie Scheler de l'autre), sur la nature des relations (Meinong et les philosophes de Graz d'un côté, Marty et les philosophes de Prague de l'autre, et Stumpf et les philosophes de Berlin quelque part entre eux), sur la perception des phénomènes temporellement étendus (Brentano d'un côté, Meinong et Ehrenfels de l'autre, et Husserl quelque part ailleurs), sur la nature du jugement (Brentano d'un côté, Meinong et Husserl de l'autre, et Reinach quelque part ailleurs), sur la nature de la perception interne (si une telle chose existe: Brentano d'un côté, Husserl de l'autre, et Geiger et Scheler quelque part ailleurs), ou encore sur la nature de la vérité (Brentano d'un côté, Meinong, Husserl le Jeune et Husserl le Vieux de l'autre).

Si Mulligan ne nie pas l'existence de ces conflits, il leur donne toutefois un rôle relativement secondaire en ce que ceux-ci ne semblent pas affecter sérieusement l'unité du courant philosophique qui, elle, est assurée par le système de vérités essentielles. Cela implique de minimiser la portée de certains conflits. Dans le cas justement du conflit sur la nature de la vérité - comme rectitude (Richtigkeit) du jugement chez Brentano ou correspondance d'une proposition avec un état de choses chez Husserl - (SM) appuyée par la version forte de $(\mathrm{TM})$ et $(\mathrm{HM})$ inviterait à penser que lorsque Brentano affirme:

La vérité appartient au jugement de celui qui juge correctement [dem Urteile des richtig Urteilenden zukommt], c'est-à-dire au jugement de celui qui juge comme quelqu'un qui, jugeant la même chose, jugerait avec évidences ${ }^{8}$.

8. Brentano (I930), p. I39. Ici et dans ce qui suit, je m'en tiens à la conception épistémique de la vérité défendue par Brentano. Cette conception n'est pas la seule, Brentano ayant 
Il exprimerait une vérité essentielle qui "peut servir» (Husserl) ou "peut fonctionner» (Mulligan) comme une norme (ce que jugerait quelqu'un qui jugerait avec évidence), sans toutefois en être une. Or il me semble que le débat austro-allemand sur le psychologisme qui oppose notamment Brentano à Husserl est en grande partie basé sur le refus de (HM) par plusieurs Brentaniens. Dans son introduction à la Psychologie de Brentano de I924, Kraus rejette la distinction introduite par $(\mathrm{HM})$ :

Cela ne fait aucun sens de débattre de la question de savoir si on doit appeler le jugement évident, c'est-à-dire le jugement justifié en soi, une «norme» ou bien seulement quelque chose qui fonde une norme'.

Kraus fait cette affirmation parce qu'il croit, avec Brentano, que l'évidence d'un jugement fonde sa vérité. La réplique de Brentano et de Kraus à (HM) est univoque:

Les lois [Gebote] de la logique sont naturellement des règles valides du juger, c'est-à-dire qu'on doit s'en remettre à elles parce que le juger qui est conforme à ces règles est sûr, et que celui qui déroge à ces règles donne accès à l'erreur; il s'agit donc d'une préférence naturelle du processus de pensée conforme aux règles face à celui qui s'y oppose ${ }^{10}$.

Tout jugement évident est un jugement pleinement correct; c'est ce qui est en soi justifié ou qui se justifie en soi, le jugement tel qu'il doit être; pour cette raison, c'est quelque chose qui donne la direction, qui donne la norme, quelque chose de normatif; on pourrait aussi l'appeler le «jugement idéal », car il réalise l'idéal logique, à savoir la correction [Korrektheit], la rectitude [Richtigkeit $[$ [...] dans tout jugement évident, le devoir-être logique devient un être logique $[\ldots]$ quelque chose peut très bien être comme ce doit être. Et on doit donc partir du jugement évident [...] celui-ci est normatif dans un autre sens que celui des énoncés qui nous incitent à faire ceci ou cela afin de parvenir à des jugements corrects; il est normatif dans le sens où il est lui-même un jugement idéal et nous dit: "c'est ainsi et pas autrement que tu dois juger si tu veux juger correctement ${ }^{11}$ ».

Que Brentano et Kraus rejettent (HM) n'est pas en soi un motif empêchant d'interpréter la thèse brentanienne que la vérité appartient au jugement de celui qui juge correctement simplement comme étant l'emploi normatif d'une vérité essentielle. Toutefois, les trois dernières citations ne s'attaquent pas seulement à (HM), mais aussi à sa version élargie, (HM*). En rejetant

défendu à certains moments une conception correspondantiste introduisant des entités abstraites (les "contenus de jugement").

9. Kraus (I924), p. LXI.

10. Brentano (I955), p. I2.

11. Kraus, ibid. Ce passage est clairement inspiré d'une lettre de Brentano adressée à Kraus en I9I6, telle que reproduite dans Brentano (I966), p. 300: "Un jugement qui est tel qu'il doit être est un jugement qui correspond à la règle logique. Et c'est ce que fait le jugement évident, aussi bien le jugement immédiatement que le jugement médiatement évident. » 
(5) dans (HM*) - et en acceptant à l'inverse que (2) fonde (I), Brentano et Kraus mettent à mal au moins la version forte de $(\mathrm{TM})$, mais aussi $(\mathrm{HM})^{12}$. Du coup, elles semblent poser un problème pour $(\mathrm{SM})$ : elles suggèrent qu'au moins certaines des vérités essentielles décrites dans la philosophie austroallemande non seulement fonctionnent comme des normes, mais sont des normes. En ce sens, on peut appliquer (HM) pour déterminer un facteur unifiant pour au moins un ou plusieurs courants de la philosophie austroallemande, mais le recours à d'autres facteurs, externes, semble inévitable: par exemple l'attachement historique à une maxime - Vera philosophiae methodus nulla alia nisi scientiae naturalis est — l'intention de Brentano de fonder une école, ou encore certaines allégeances scolastiques peuvent être prises en considération pour compléter (HM), si on veut rendre compte de certains éléments discordants par rapport à ce principe au sein de la philosophie austro-allemande ${ }^{13}$.

\section{L'essence du langage et la signification}

Le chapitre VI de l'ouvrage vise à étayer (TM) en se penchant tout particulièrement sur les thèses concernant le langage. Ici, deux courants sont distingués: celui qui définit la signification comme ce que nous faisons avec les mots, et celui qui conçoit la signification comme ce que les mots font (indépendamment de nous). Ces deux courants représentent deux tendances parmi les héritiers de Brentano: le premier est représenté notamment par Marty, Martinak, Ahlman, et Bühler et suit l'idée que le langage est une structure empirico-téléologique. Le second est représenté principalement par Husserl qui, inspiré par Bolzano, voit les fonctions de signification comme déterminées par et dépendantes des structures formelles des propositions. En partant de cette distinction, Mulligan veut montrer comment le prétendu rejet par Wittgenstein d'une «essence du langage» dans les Investigations masque en fait des ressemblances frappantes entre ses propres observations et celles émises notamment par Bühler sur l'essence du langage, suggérant que les considérations sur le langage des Investigations constituent une contribution au programme de Brentano.

Les éléments avancés par Mulligan pour étayer (TM) dans ce chapitre sont convaincants. En effet, même si Bühler interprète le langage comme un phénomène biologique et sociologique, fondé sur un dressage aveugle, et que ses analyses sur le guidage sont largement inspirées de la biologie et de la psychologie comportementale et développementale, il ne tombe pas dans le biologisme ou le sociologisme, comme le remarque Mulligan, puisque le

12. Le rejet de (HM) et (HM*) par Brentano et Kraus porte en fait aussi sur $\left(\mathrm{HM}^{*} \mathrm{e}\right)$ car, pour eux, (2) fonde (I) aussi bien pour les vérités logiques (dans ce cas, il s'agit de l'évidence des axiomes) que pour les vérités essentielles (dans ce deuxième cas, il s'agit de l'évidence de la perception interne).

13. Sur ces facteurs externes, voir Fisette et Fréchette (2007). 
langage est régi par ce que Bühler appelle des lois structurelles (Strukturgesetze), qui elles n'ont aucun caractère normatif. En effet, les lois structurelles du Bühler de la Sprachtheorie ne sont pas des régularités empiriques, mais plutôt des lois d'essences à considérer dans le sens des Wesensgesetze de Husserl dans les Recherches logiques.

Puisque Bühler et Marty sont identifiés au même courant dans ce chapitre, Mulligan serait probablement d'accord avec une description de la théorie martyienne de la signification en des termes un peu semblables à ceux de la théorie bühlérienne, et qui du coup illustrerait également (HM), ou plus précisément un principe comme $\left(\mathrm{HM}^{*} \mathrm{e}\right)$ portant sur les vérités essentielles. Pour Marty, avoir une signification pour une expression est déterminé par l'usage de ceux qui emploient cette expression - en disant "Philippe était le père d'Alexandre de Macédoine», je veux inviter mon interlocuteur à former un jugement similaire au mien, mais je veux aussi manifester un contenu en particulier, l'état de choses exprimé par la proposition énoncée. La composante normative est évidente et elle est manifeste dans l'invitation adressée à mon interlocuteur à former un jugement. Or on pourrait interpréter Marty ici dans le sens de (HM) et dire qu'un acte de signification peut fonctionner comme une règle, mais que le «moment normatif» inclus en lui dépend de quelque chose de non normatif, dans ce cas, de la vérité exprimée par la proposition énoncée. Cela reviendrait à dire que lorsque je dis à mon interlocuteur (en exprimant quelque chose que je pense vraiment):

(I) «Philippe était le père d'Alexandre de Macédoine »,

mon énoncé «fonctionne comme une règle », qui serait la suivante:

(2) «Tu dois penser que Philippe était le père d'Alexandre de Macédoine.»

où la rectitude de la règle exprimée en (2) dépend de la rectitude de (I), et où la rectitude de (I) n'est pas normative.

Une telle lecture de la théorie martyienne de la signification est probablement à favoriser si on veut utiliser (HM) et la version forte de (TM) pour avancer (SM). Mais elle suppose toutefois que l'état de choses exprimé par la proposition énoncée a une indépendance ontologique visà-vis de l'acte de signification si cet état de choses, suivant (HM), est censé fonder le moment normatif de l'acte de signification. Or la question de l'indépendance ontologique des Gebilde (Stumpf) ou des Sachverhalte (Marty) ne me semble pas suffisamment univoque pour appuyer la lecture suggérée: c'est d'ailleurs justement un peu en réaction aux accusations de psychologisme formulées par Husserl dans les Prolégomènes cités plus haut que d'autres héritiers de Brentano, comme Stumpf, Marty ou Twardowski, ont ressenti le besoin de se distancier du psychologisme dans plusieurs écrits du début du siècle. Il n'est pas clair que les résultats de cette manœuvre, qu'Ingarden a déjà décrite comme la «tentative de se frayer un 
chemin entre Scylla et Charybde ${ }^{14}$ » soient suffisants: Stumpf (I906) considère les Gebilde comme indépendants des actes individuels de signifier, mais nécessairement corrélatifs à un tel acte. De manière similaire, Marty leur attribue une certaine indépendance, tout en spécifiant qu'il n'y a d'états de choses que pour les jugements vrais, et que ceux-ci n'ont d'existence que tant qu'ils sont corrélatifs d'un acte de jugement correspondant ${ }^{15}$.

Si des théories des états de choses comme celles de Stumpf et Marty, qui font de ceux-ci en quelque sorte des produits ${ }^{16}$ de nos activités cognitives, sont à voir éventuellement comme des illustrations de (HM), il faudrait admettre que (HM), tant dans sa version portant sur les vérités logiques que celle portant sur les vérités essentielles, est compatible avec un principe qu'on peut tirer des trois citations de Brentano et Kraus et qu'on pourrait appeler (BK):

I) $(\mathrm{p} \&(\mathrm{p} \rightarrow \mathrm{q})) \rightarrow \mathrm{q}$

2) si $(\mathrm{p} \&(\mathrm{p} \rightarrow \mathrm{q}))$, alors on a le droit d'inférer à $\mathrm{q}$

3) si (2), alors (2) en partie parce que (I)

4) si (I), alors (I) en partie parce que quelqu'un juge avec évidence ou pourrait juger (I) avec évidence

Dans le cas des vérités essentielles, disons (BKe):

I) tout ce qui est coloré est étendu

2) si $x$ est coloré, alors on a le droit d'inférer que $x$ est étendu

3) si (2), alors (2) en partie parce que (I)

4) si (I), alors (I) en partie parce que quelqu'un juge avec évidence ou pourrait juger (I) avec évidence

Cependant, je doute fort de la sympathie de Mulligan à l'égard de (BK) ou (BKe). Il me semble clair par ailleurs que ni Stumpf ni Marty (et ni Brentano, ni Kraus, etc.) ne seraient prêts à accepter que la vérité de (I) est indépendante d'un acte (potentiel ou réel) de juger (I) avec évidence. Je serais plutôt porté à dire que l'idée derrière (BK) et (BKe) est la même que celle qu'on retrouve dans les théories des états de choses de Marty, des Gebilde de Stumpf, et des produits de Twardowski, à savoir que la possibilité pour une loi d'essence d'être instanciée, ou encore la possibilité pour une loi d'essence d'être évidente - en d'autres termes, les relations exprimées par les prédicats "peut être instanciée par _ " et "peut être jugée avec évidence» — sont considérées par ces derniers comme des relations internes. En ce sens, contrairement à Husserl, Twardowski, Marty et Stumpf diraient plutôt que

14. Ingarden (I948), p. 28.

15. Marty (I908), p. $328 s q$.

16. Comparer également Twardowski (I996) / (2007). 
les lois d'essences sont constitutivement instanciées par les cas concrets (les actes mentaux correspondants).

Dans la Krise der Psychologie, et inspiré par Husserl, Bühler suggérait que ses Strukturgesetze pouvaient surmonter le problème de l'objectivité des Gebilde ${ }^{17}$. Or ces Strukturgesetze me semblent plus proches du projet husserlien que des solutions proposées par Twardowski, Marty et Stumpf. Si Mulligan a raison de suggérer que Bühler avance une conception de l'essence du langage particulièrement apte à étayer $(\mathrm{TM})$ et $(\mathrm{SM})$, et ce malgré quelques moments discordants ${ }^{18}$, on peut penser que c'est surtout en raison de sa sympathie au projet de Husserl, puisque plusieurs des thèses de Marty sur l'essence du langage sont en fait sympathiques à (BK) et (BKe).

\section{Remarques finales}

En bref, je doute que la conception épistémique de la vérité défendue par Brentano et plusieurs de ses héritiers - conception selon laquelle l'évidence est en dernière instance le seul moyen de déterminer la vérité d'un jugement - puisse être prise en considération à juste titre dans la reconstruction proposée par Mulligan. Je crois qu'il pourrait en être de même pour certains des autres conflits mentionnés plus haut en section 2, bien que je ne puisse m'étendre ici en détail sur cette question.

Par ailleurs, il est indéniable que la reconstruction de l'histoire de la philosophie austro-allemande proposée sur la base de l'importance accordée au principe (HM) est très fructueuse, particulièrement en ce qui a trait à la question du vouloir dire, de la certitude, des objets psychologiques et des couleurs, des points que je n'ai pas pu aborder ici. Et en ce qu'elle permet de montrer que la contribution de Wittgenstein peut être vue comme une contribution s'insérant dans l'unité de la philosophie austro-allemande, cette reconstruction invite à réviser plusieurs présuppositions historiques et théoriques sur la philosophie autrichienne à la fois en aval et en amont de Wittgenstein, non seulement celles des spécialistes du Cercle de Vienne et de ceux qui travaillent sur Brentano et son école, mais aussi celles de nombreux historiens de la philosophie analytique et de la phénoménologie, ce qui en fait un ouvrage incontournable ${ }^{19}$.

17. Bühler (I927), p. I07sq.

18. En effet, Mulligan n'éclipse pas les moments discordants dans l'œuvre de Bühler par rapport à l'interprétation qu'il en fait. D'abord, Bühler est représentationnaliste (p. I33sq). Son concept d'Ordner, de classeur, est un outil de représentation non linguistique, et on peut sérieusement se demander si la fonction représentative est possible sans ces classeurs. En tirant les conceptions husserliennes de la signification vers le nominalisme et le naturalisme, Bühler gauchit les conceptions autrichiennes du langage (p. I36). Mulligan remarque aussi que la thèse bühlerienne sur la fonction linguistique sui generis du représenter serait un mythe pour Wittgenstein.

19. Je remercie Kevin Mulligan pour ses généreuses suggestions à des versions antérieures de ce texte. 


\section{Bibliographie}

Brentano, F. Meine letzten Wünsche für Österreich, Wien, Cotta, I 895 .

-. Psychologie vom empirischen Standpunkt. Erster Band, Leipzig, Meiner, I924.

—. Wabrheit und Evidenz, Leipzig, Meiner, I930.

- Vom Ursprung sittlicher Erkenntnis, Hamburg, Meiner, I955.

- Die Abkehr vom Nichtrealen, Hamburg, Meiner, I966.

Bühler, K. Die Krise der Psychologie, Iéna, Fischer, 1927.

Fisette, D. et G. Fréchette (dir.) À l'école de Brentano, Paris, Vrin, 2007.

Husserl, E. Logische Untersuchungen. Erster Band. Prolegomena zur reinen Logik, Halle, Max Niemeyer, I 900.

—. Recherches logiques. Prolégomènes à la logique pure, trad. fr. de H. Élie, A. Kelkel et R. Scherer, Paris, PUF (trad. fr. de Husserl, I900), I959.

Ingarden, R. "The Scientific Activity of Kazimierz Twardowski », Studia Philosophica, vol. 3, 1948, p. 17-30.

Kraus, O. «Einleitung des Herausgebers », in Brentano, I924, p. Xv-xcviii.

Marty, A. Untersuchungen zur Grundlegung der allgemeinen Grammatik und Sprachphilosophie, Halle, Niemeyer, I908.

Mulligan, K. Wittgenstein et la philosophie austro-allemande, Paris, Vrin, $20 \mathrm{I} 2$.

Reinach, A. I9I4. "Was ist Phänomenologie?", in A. Reinach, Sämtliche Werke. Band 1. Die Werke, Munich, Philosophia, I989, p. 379-405.

Stumpf, C. "Erscheinung und psychische Funktionen", in Abhandlungen der Königlich-Preußischen Akademie der Wissenschaften, Philosophisch-historische Klasse, Berlin, Reimer, I906, p. 3-40.

—. "Phénomènes et fonctions psychiques", dans D. Fisette (dir.), Carl Stumpf. Renaissance de la philosophie (traduction française de Stumpf [1906]), Paris, Vrin, 2006, p. I33-I68.

Twardowski, K. "Funktionen und Gebilde» in Conceptus, vol. 29, n 75, I996, p.I 57-I 89 .

- «Fonctions et formations », in D. Fisette et G. Fréchette (traduction française de Twardowski [I996]), 2007, p. 343-383. 